\title{
A simple proof for a forbidden subposet problem
}

\author{
Ryan R. Martin* \\ Department of Mathematics \\ Iowa State University \\ Ames, IA, U.S.A. \\ rymartin@iastate.edu \\ Andrew Uzzell ${ }^{\ddagger}$
}

Department of Mathematics and Statistics

Grinnell College

Grinnell, IA, U.S.A.

uzzellan@grinnell.edu

\author{
Abhishek Methuku ${ }^{\dagger}$ \\ School of Mathematics \\ École Polytechnique Fédérale \\ Lausanne, Switzerland \\ abhishekmethuku@gmail.com \\ Shanise Walker $\S$ \\ Department of Mathematics \\ University of Wisconsin-Eau Claire \\ Eau Claire, WI, U.S.A. \\ shaniseg@uwec.edu
}

Submitted: Feb 21, 2018; Accepted: Aug 20, 2019; Published: Jan 24, 2020

(c) The authors. Released under the CC BY license (International 4.0).

\begin{abstract}
The poset $Y_{k, 2}$ consists of $k+2$ distinct elements $x_{1}, x_{2}, \ldots, x_{k}, y_{1}, y_{2}$, such that $x_{1} \leqslant x_{2} \leqslant \ldots \leqslant x_{k} \leqslant y_{1}, y_{2}$. The poset $Y_{k, 2}^{\prime}$ is the dual poset of $Y_{k, 2}$. The sum of the $k$ largest binomial coefficients of order $n$ is denoted by $\Sigma(n, k)$. Let $\operatorname{La}^{\sharp}\left(n,\left\{Y_{k, 2}, Y_{k, 2}^{\prime}\right\}\right)$ be the size of the largest family $\mathcal{F} \subset 2^{[n]}$ that contains neither $Y_{k, 2}$ nor $Y_{k, 2}^{\prime}$ as an induced subposet. Methuku and Tompkins proved that $\operatorname{La}^{\sharp}\left(n,\left\{Y_{2,2}, Y_{2,2}^{\prime}\right\}\right)=\Sigma(n, 2)$ for $n \geqslant 3$ and conjectured the generalization that if $k \geqslant$ 2 is an integer and $n \geqslant k+1$, then $\mathrm{La}^{\sharp}\left(n,\left\{Y_{k, 2}, Y_{k, 2}^{\prime}\right\}\right)=\Sigma(n, k)$. On the other hand, it is known that $\mathrm{La}^{\sharp}\left(n, Y_{k, 2}\right)$ and $\mathrm{La}^{\sharp}\left(n, Y_{k, 2}^{\prime}\right)$ are both strictly greater than $\Sigma(n, k)$. In this paper, we introduce a simple approach, motivated by discharging, to prove this conjecture.
\end{abstract}

Mathematics Subject Classifications: 06A06

*Research supported in part by a Simons Foundation grant (\# 353292) and by NSF-DMS Grants $1604458,1604773,1604697$, and 1603823 .

${ }^{\dagger}$ Research supported in part by the Hungarian National Research, Development and Innovation Office - NKFIH under the grant K116769 and by a generous grant from the Combinatorics Foundation.

${ }^{\ddagger}$ Research supported in part by a generous grant from the Institute for Mathematics and its Applications and in part by NSF-DMS Grants 1604458, 1604773, 1604697, and 1603823.

${ }^{\S}$ Research supported in part by NSF-DMS Grants 1604458, 1604773, 1604697, and 1603823. 


\section{Introduction}

The $n$-dimensional Boolean lattice, denoted $\mathcal{B}_{n}$, is the partially ordered set (poset) $\left(2^{[n]}, \subseteq\right)$, where $[n]=\{1, \ldots, n\}$. For any $0 \leqslant i \leqslant n$, let $\left(\begin{array}{c}{[n]} \\ i\end{array}\right):=\{A \subseteq[n]:|A|=i\}$ denote the $i$ th level of the Boolean lattice. Let $P$ be a finite poset and $\mathcal{F}$ be a family of subsets of $[n]$. We say that $P$ is contained in $\mathcal{F}$ as a weak subposet if there is an injection $\alpha: P \rightarrow \mathcal{F}$ satisfying $x_{1}<_{p} x_{2} \Longrightarrow \alpha\left(x_{1}\right) \subset \alpha\left(x_{2}\right)$ for all $x_{1}, x_{2} \in P$. $\mathcal{F}$ is called $P$-free if $P$ is not contained in $\mathcal{F}$ as a weak subposet. We define the corresponding extremal function to be $\operatorname{La}(n, P):=\max \{|\mathcal{F}|: \mathcal{F}$ is $P$-free $\}$. Analogously, if $P, Q$ are two posets, then $\mathrm{La}(n,\{P, Q\}):=\max \{|\mathcal{F}|: \mathcal{F}$ is $P$-free and $Q$-free $\}$.

The linearly ordered poset on $k$ elements, $a_{1}<a_{2}<\cdots<a_{k}$, is called a chain of length $k$, and is denoted by $P_{k}$. Using this notation the well-known theorem of Sperner [15] can be stated as $\mathrm{La}\left(n, P_{2}\right)=\left(\begin{array}{c}n \\ \lfloor n / 2\rfloor\end{array}\right)$. Let us denote the sum of the $k$ largest binomial coefficients of order $n$ by $\Sigma(n, k)$. Erdős [6] extended Sperner's theorem by showing that $\mathrm{La}\left(n, P_{k}\right)=\Sigma(n, k-1)$, with equality if and only if the family is the union of $k-1$ largest levels of the Boolean lattice. Notice that, since any poset $P$ is a weak subposet of a chain of length $|P|$, Erdős's theorem implies that $\mathrm{La}(n, P) \leqslant(|P|-1)\left(\begin{array}{c}n \\ \lfloor n / 2\rfloor\end{array}\right)=O\left(\left(\begin{array}{c}n \\ \lfloor n / 2\rfloor\end{array}\right)\right)$. Later many authors, including Katona and Tarján [12], Griggs and Lu [9], and Griggs, Li, and $\mathrm{Lu}[8]$ studied $\mathrm{La}(n, P)$ for various other posets $P$. (See the recent survey by Griggs and $\mathrm{Li}$ [7] for an excellent survey of all the posets that have been studied.) Let $h(P)$ denote the height (maximum length of a chain) of $P$. One of the first general results is due to Bukh [2], who showed that if $T$ is a finite poset whose Hasse diagram is a tree of height $h(T) \geqslant 2$, then $\operatorname{La}(n, T)=(h(T)-1+O(1 / n))\left(\begin{array}{c}n \\ |n / 2|\end{array}\right)$. The most notorious poset for which the asymptotic value of the extremal function is still unknown is the diamond $Q_{2}$, the poset on 4 elements with the relations $a<b, c<d$ where $b$ and $c$ are incomparable. The best known bound is $(2.20711+o(1))\left(\begin{array}{c}n \\ \lfloor n / 2\rfloor\end{array}\right)$, due to Grósz, Methuku, and Tompkins [10].

We say that $P$ is contained in $\mathcal{F}$ as an induced subposet if and only if there is an injection $\alpha: P \rightarrow \mathcal{F}$ satisfying $x_{1}<_{p} x_{2} \Longleftrightarrow \alpha\left(x_{1}\right) \subset \alpha\left(x_{2}\right)$ for all $x_{1}, x_{2} \in P$. We say that $\mathcal{F}$ is induced-P-free if $P$ is not contained in $\mathcal{F}$ as an induced subposet. We define the corresponding extremal function as $\operatorname{La}^{\sharp}(n, P):=\max \{|\mathcal{F}|: \mathcal{F}$ is induced $P$-free $\}$. Analogously, if $P, Q$ are two posets, then

$$
\mathrm{La}^{\sharp}(n,\{P, Q\}):=\max \{|\mathcal{F}|: \mathcal{F} \text { is induced } P \text {-free and induced } Q \text {-free }\} .
$$

Despite the considerable progress that has been made on the extremal function for forbidden weak subposets, little is known about forbidden induced subposets (except for $P_{k}$, where the weak and induced containment are equivalent). The first results for forbidden induced subposets are due to Carroll and Katona [3] and to Katona [11], showing $\mathrm{La}^{\sharp}\left(n, V_{r}\right)=(1+o(1))\left(\begin{array}{c}n \\ \lfloor n / 2\rfloor\end{array}\right)$ where $V_{r}$ is the $r$-fork poset $\left(x \leqslant y_{i}\right.$ for all $\left.1 \leqslant i \leqslant r\right)$.

Boehnlein and Jiang [1] generalized the results of $[3,11]$ by extending Bukh's result to induced containment of tree-shaped posets, $T$, proving La $\sharp(n, T)=(h(T)-1+o(1))\left(\begin{array}{c}n \\ \lfloor n / 2\rfloor\end{array}\right)$. Only recently, Methuku and Pálvölgyi [14] showed that for every poset $P$, there is a constant $c_{P}$ depending only on $P$ such that $\operatorname{La}^{\sharp}(n, P) \leqslant c_{P}\left(\begin{array}{c}n \\ \lfloor n / 2\rfloor\end{array}\right)$. 
Even fewer exact results are known for forbidden induced subposets, which is the topic of this paper. Katona and Tarján [12] proved that La $(n,\{V, \Lambda\})=\operatorname{La}^{\sharp}(n,\{V, \Lambda\})=$ $2\left(\begin{array}{c}n-1 \\ \left\lfloor\frac{n-1}{2}\right\rfloor\end{array}\right)$, where $V$ and $\Lambda$ are the 2 -fork and its dual, the 2-brush, respectively.

Now we formally define the posets considered in this paper.

Definition 1. Let $k, r \geqslant 2$ be integers. The $r$-fork with a $k$-shaft poset consists of $k+r$ elements $x_{1}, x_{2}, \ldots, x_{k}, y_{1}, y_{2}, \ldots, y_{r-1}, y_{r}$ with $x_{1} \leqslant x_{2} \leqslant \cdots \leqslant x_{k}$ and $x_{k} \leqslant y_{i}$ for all $1 \leqslant i \leqslant r$, and is denoted by $Y_{k, r}$. Let $Y_{k, r}^{\prime}$ denote the reversed poset of $Y_{k, r}$, also called the dual poset of $Y_{k, r}$.

For simplicity, we will write $Y_{k}$ and $Y_{k}^{\prime}$ instead of $Y_{k, 2}$ and $Y_{k, 2}^{\prime}$ respectively.

The first result about $Y_{k, r}$ was due to Thanh [16] who showed that $\operatorname{La}\left(n, Y_{k, r}\right)=$ $(k+o(1))\left(\begin{array}{c}n \\ \lfloor n / 2\rfloor\end{array}\right)$. The lower order term in his upper bound was improved by De Bonis and Katona [4]. Thanh also gave a construction showing that $\mathrm{La}\left(n, Y_{k, r}\right)>\Sigma(n, k)$. Methuku and Tompkins [13] showed that if one forbids both $Y_{k}$ and $Y_{k}^{\prime}$, then an exact result can be obtained: $\operatorname{La}\left(n,\left\{Y_{k}, Y_{k}^{\prime}\right\}\right)=\Sigma(n, k)$.

Using a cycle decomposition method, they also showed the following exact result for induced posets.

Theorem 2 (Methuku-Tompkins [13]). If $n \geqslant 3$, then $\mathrm{La}^{\sharp}\left(n,\left\{Y_{2}, Y_{2}^{\prime}\right\}\right)=\Sigma(n, 2)$.

Theorem 2 strengthens the result of De Bonis, Katona, and Swanepoel [5] stating that $\mathrm{La}(n, B)=\Sigma(n, 2)$ where $B$ is the butterfly poset which consists of 4 elements $a, b, c, d$ with $a, b \leqslant c, d$. Indeed if a family does not contain the butterfly as a subposet, then it contains neither $Y_{2}$ nor $Y_{2}^{\prime}$ as an induced subposet. However, a family might contain neither an induced $Y_{2}$ nor an induced $Y_{2}^{\prime}$ while still containing a butterfly.

In Section 3, we establish the following generalization of Theorem 2 by proving a conjecture from [13].

Theorem 3. If $k \geqslant 2$ is an integer and $n \geqslant k+1$, then $\operatorname{La}^{\sharp}\left(n,\left\{Y_{k}, Y_{k}^{\prime}\right\}\right)=\Sigma(n, k)$.

Note that forbidding only one of $Y_{k}$ and $Y_{k}^{\prime}$ is not enough to obtain an exact result. Indeed, again by Thanh's construction [16] we have $\mathrm{La}^{\sharp}\left(n, Y_{k}\right)>\Sigma(n, k)$ and $\mathrm{La}^{\sharp}\left(n, Y_{k}^{\prime}\right)>$ $\Sigma(n, k)$.

In the course of proving Theorem 3, we establish the following LYM-type inequality in the case that $\varnothing$ and $[n]$ are not in our family.

Theorem 4. Let $k \geqslant 2$ be an integer and $n \geqslant k+1$. If $\mathcal{F} \subset 2^{[n]}$ contains neither $Y_{k}$ nor $Y_{k}^{\prime}$ as an induced subposet and $\varnothing,[n] \notin \mathcal{F}$, then

$$
\sum_{F \in \mathcal{F}}\left(\begin{array}{c}
n \\
|F|
\end{array}\right)^{-1} \leqslant k .
$$

In order to prove Theorem 4 we use a double-counting argument that is reminiscent of discharging-type arguments, as described in the next section. We then prove Theorem 3 by using Theorem 4 and induction on $k$. 


\section{Preliminaries and our approach}

The following terminology will be used to prove Theorems 3 and 4 . Let $\mathcal{F}$ be a family of subsets of $[n]$ which is induced $Y_{k}$-free and induced $Y_{k}^{\prime}$-free. For sets $U, V \subseteq[n]$, let the interval $[U, V]$ denote the Boolean lattice induced by the collection of all sets that contain $U$ and are contained in $V$. A chain $C$ where $C=\left\{A_{0}, \ldots, A_{n}\right\}$ and $\varnothing=A_{0} \subset A_{1} \subset \cdots \subset$ $A_{n}=[n]$ is called a full chain or a maximal chain.

A spine is a chain $A_{1} \subset A_{2} \subset \cdots \subset A_{\ell}$ such that $\left|A_{i+1} \backslash A_{i}\right|=1$ for $1 \leqslant i \leqslant \ell-1$ where there are exactly $k-1$ members of $\mathcal{F}$ in $\left\{A_{1}, \ldots, A_{\ell}\right\}$ and where $A_{1}, A_{\ell} \in \mathcal{F}$. Note that a spine may contain elements not from $\mathcal{F}$.

Let $\mathcal{C}$ be the set of all full chains and let $\mathcal{S}$ be the set of all spines. We say that a full chain $C \in \mathcal{C}$ is associated with a spine $S \in \mathcal{S}$ or that $C$ contains $S$ as a spine if either

1. $C$ has exactly $k-1$ members of $\mathcal{F}$, which we name $F_{1}, \ldots, F_{k-1}$. In this case, $C$ is associated with the spine that is a subchain of $C$ from $F_{1}$ to $F_{k-1}$; or

2. $C$ has exactly $k+x$ elements of $\mathcal{F}$ (where $x \geqslant 1$ ), which we name $F_{1}, \ldots, F_{k+x}$. In this case, $C$ is associated with $x$ spines, namely $S_{F_{i}}$ for $2 \leqslant i \leqslant x+1$, where $S_{F_{i}}$ is the spine that is a subchain of $C$ from $F_{i}$ to $F_{i+k-2}$. (Notice that a chain $C$ with at least $k+1$ elements of $\mathcal{F}$ is not associated with the spines that correspond to the first $k-1$ elements of $\mathcal{F} \cap C$ and to the last $k-1$ elements of $\mathcal{F} \cap C$.)

Let spine $(C)$ denote the set of all spines that $C$ contains. More precisely,

$$
\text { spine }(C):=\{S: C \text { contains } S \text { as a spine }\} \text {. }
$$

We start by placing a weight (or charge) on a spine depending on the chains that contain it. More precisely, if $S \in \mathcal{S}$ is a spine and $C \in \mathcal{C}$ is a full chain, then we define a weight function $w(S, C)$ as follows.

$$
w(S, C)= \begin{cases}1, & \text { if } S \in \operatorname{spine}(C) \text { and } C \text { contains at least } k+1 \text { members of } \mathcal{F} \\ -1, & \text { if } S \in \operatorname{spine}(C) \text { and } C \text { contains exactly } k-1 \text { members of } \mathcal{F}\end{cases}
$$

The motivation behind the above weight function is that, when considering all the chains $C$ associated with a fixed spine $S$, we would like to show that the total positive charge of these chains is outweighed by their total negative charge. This is confirmed by Lemma 6 . Moreover, notice that for any $C \in \mathcal{C}$ with spine $(C) \neq \varnothing$, we have the following useful identity:

$$
\sum_{\substack{S \in \mathcal{S} \\ S \in \operatorname{spine}(C)}} w(S, C)=|\mathcal{F} \cap C|-k .
$$

Observe that any chain $C$ having exactly $k-1$ or at least $k+1$ members of $\mathcal{F}$ must contain a spine $S$. It follows that if $\operatorname{spine}(C)=\varnothing$ then $C$ contains at most $k$ members of $\mathcal{F}$. In other words, 


$$
\sum_{\substack{C \in \mathcal{C} \\ \operatorname{spine}(C)=\varnothing}}(|\mathcal{F} \cap C|-k) \leqslant 0 .
$$

Before proving Lemma 6, we need the following straightforward counting lemma, the proof of which we provide for completeness.

Lemma 5. Let $n \geqslant 2$. If $\mathcal{G} \subseteq\{\{1\},\{1,2\},\{1,2,3\}, \ldots,\{1,2,3 \ldots, n-1\}\}$, then the number of full chains in $2^{[n]}$ containing no member of $\mathcal{G}$ is at least the number of full chains that contain at least one member of $\mathcal{G}$.

Proof. Let the set of full chains that contain at least one member of $\mathcal{G}$ be $X$ and the set of full chains that contain no member of $\mathcal{G}$ be $Y$. To show that $|X| \leqslant|Y|$ we will construct an injection from $X$ to $Y$. Consider any chain $C \in X$. Let $C$ be $\varnothing \subset\left\{x_{1}\right\} \subset\left\{x_{1}, x_{2}\right\} \subset$ $\left\{x_{1}, x_{2}, x_{3}\right\} \subset \cdots \subset\left\{x_{1}, x_{2}, x_{3}, \ldots, x_{n}\right\}$. For simplicity, we will say the permutation corresponding to $C$ is $x_{1} x_{2} x_{3} \cdots x_{n}$.

If $\left\{x_{1}, x_{2}, \ldots, x_{j}\right\}$ is the last set from $\mathcal{G}$ in $C$ and $x_{i}=1$, then $x_{1} x_{2} \cdots x_{j}$ is a permutation of $\{1,2, \ldots, j\}$. Hence, $x_{j+1} \geqslant j+1$ and $1 \leqslant i \leqslant j$. Let us consider the chain $C^{\prime}$ corresponding to the permutation

$$
x_{1} x_{2} \cdots x_{i-1} x_{j+1} x_{i+1} x_{i+2} \cdots x_{j} x_{i} x_{j+2} \cdots x_{n},
$$

obtained by swapping $x_{j+1}$ with $x_{i}$ in the permutation corresponding to $C$. If $C^{\prime}$ contains the set $\{1,2, \ldots, j+1\}$, then it must be the case that $x_{j+1}=j+1$. Thus, $C$ contains the set $\{1,2, \ldots, j+1\}$, which contradicts the maximality of $j$. Therefore, under this map, the full chain $C^{\prime}$ does not contain any member of $\mathcal{G}$. If we map $C \in X$ to $C^{\prime} \in Y$ in this way, the map is an injection, as desired.

Lemma 6. Let $k \geqslant 2$ be an integer and $n \geqslant k+1$. Let $\mathcal{F}$ be a family in $\mathcal{B}_{n}$ with no induced $Y_{k}$ and no induced $Y_{k}^{\prime}$ such that $\varnothing,[n] \notin \mathcal{F}$. Let $\mathcal{S}$ denote the set of spines of $\mathcal{F}$ and let $\mathcal{C}$ denote the set of full chains in $\mathcal{B}_{n}$. For any $S \in \mathcal{S}$,

$$
\sum_{\substack{C \in \mathcal{C} \\ S \in \operatorname{spine}(C)}} w(S, C) \leqslant 0 .
$$

Proof. Let a spine $S$ be the chain $A_{1} \subset A_{2} \subset \cdots \subset A_{\ell}$ where $\left|A_{i+1} \backslash A_{i}\right|=1$ for $1 \leqslant i \leqslant \ell-1$. (Recall that, by definition of a spine, there are exactly $k-1$ members of $\mathcal{F}$ in $\left\{A_{1}, \ldots, A_{\ell}\right\}$ and $A_{1}, A_{\ell} \in \mathcal{F}$.) If all of the chains $C \in \mathcal{C}$ that contain $S$ as a spine have at most $k$ members of $\mathcal{F}$ then since $w(S, C)=-1$ for each of these chains, our lemma follows trivially. Therefore, we may assume that there is a chain $C \in \mathcal{C}$ that contains $S$ as a spine such that $w(S, C)=1$. By definition (of $w(S, C)=1$ ), such a chain $C$ has at least $k+1$ members of $\mathcal{F}$. Moreover, $C$ must have sets $P, Q \in \mathcal{F}$ with $P \subset A_{1}$ and $A_{\ell} \subset Q$.

If two sets $A, B \in \mathcal{F}$ are unrelated to each other and $A, B \subset A_{1}$ then we have an induced copy of $Y_{k}^{\prime}$ consisting of $A, B$, the $k-1$ members of $\mathcal{F}$ in $S$, and $Q$. Therefore, 
$\mathcal{F} \cap\left[\varnothing, A_{1}\right]$ induces a chain $\mathcal{G}_{1}$. By symmetry, $\mathcal{F} \cap\left[A_{\ell},[n]\right]$ induces a chain $\mathcal{G}_{2}$ as well. By assumption, $\varnothing,[n] \notin \mathcal{F}$, so the chains $\mathcal{G}_{1} \backslash\left\{A_{1}\right\}$ and $\mathcal{G}_{2} \backslash\left\{A_{\ell}\right\}$ may be extended to chains that satisfy the hypotheses of Lemma 5 for $\left[\varnothing, A_{1}\right]$ and $\left[A_{\ell},[n]\right]$.

Therefore, the number $a_{0}$ of full chains in $\left[\varnothing, A_{1}\right]$ containing no member of $\mathcal{G}_{1} \backslash\left\{A_{1}\right\}$ is at least the number $a_{1}$ of full chains in $\left[\varnothing, A_{1}\right]$ that contain a member of $\mathcal{G}_{1} \backslash\left\{A_{1}\right\}$. Similarly, the number $b_{0}$ of full chains in $\left[A_{\ell},[n]\right]$ containing no member of $\mathcal{G}_{2} \backslash\left\{A_{\ell}\right\}$ is at least the number $b_{1}$ of full chains in $\left[A_{\ell},[n]\right]$ that contain a member of $\mathcal{G}_{2} \backslash\left\{A_{\ell}\right\}$. Now notice that the number of chains $C \in \mathcal{C}$ associated with the spine $S$ that have exactly $k-1$ members of $\mathcal{F}$ is $a_{0} \cdot b_{0}$ and the number of chains $C \in \mathcal{C}$ associated with $S$ that have at least $k+1$ members of $\mathcal{F}$ is $a_{1} \cdot b_{1}$ (here we used that $S$ is not associated with such a chain $C$ unless $C$ has a member of $\mathcal{F}$ both above and below $S$ ). Therefore, since $a_{1} \leqslant a_{0}$ and $b_{1} \leqslant b_{0}$,

$$
\sum_{\substack{C \in \mathcal{C} \\ S \in \operatorname{spine}(C)}} w(S, C)=a_{1} \cdot b_{1}-a_{0} \cdot b_{0} \leqslant 0
$$

\section{Proofs of Theorem 3 and Theorem 4}

Recall that $\Sigma(n, k)$ denotes the sum of the sizes of the largest $k$ levels in the Boolean lattice $\mathcal{B}_{n}$. First we use a folklore lemma that establishes an inequality very similar to the LYM inequality.

Lemma 7 (See [17, Lemma 1]). If $\mathcal{F} \subseteq 2^{[n]}$ satisfies

$$
\sum_{F \in \mathcal{F}}\left(\begin{array}{c}
n \\
|F|
\end{array}\right)^{-1} \leqslant k
$$

then $|\mathcal{F}| \leqslant \Sigma(n, k)$.

The above lemma can be proved easily: if we fix $|\mathcal{F}|$, the left-hand side of (3) is minimized when we choose the subsets with sizes as near to $n / 2$ as possible. (See [17] for a more detailed proof.)

Proof of Theorem 4. Observe that by Lemma 6 ,

$$
\sum_{S \in \mathcal{S}} \sum_{\substack{C \in \mathcal{C} \\ \text { spine }(C) \ni S}} w(S, C) \leqslant 0
$$

Now by double counting,

$$
\sum_{S \in \mathcal{S}} \sum_{\substack{C \in \mathcal{C} \\ \text { spine }(C) \ni S}} w(S, C)=\sum_{\substack{C \in \mathcal{C} \\ \text { spine }(C) \neq \varnothing}} \sum_{S \in \operatorname{spine}(C)} w(S, C) .
$$

Recall that for any $C \in \mathcal{C}$ with spine $(C) \neq \varnothing$, the identity (1) holds. 
So by (4) and (5), we have

$$
\sum_{\substack{C \in \mathcal{C} \\ \operatorname{spine}(C) \neq \varnothing}}(|\mathcal{F} \cap C|-k) \leqslant 0
$$

Adding up (2) and (6), we get

$$
\sum_{C \in \mathcal{C}}(|\mathcal{F} \cap C|-k) \leqslant 0
$$

On the other hand, $\sum_{C \in \mathcal{C}}(|\mathcal{F} \cap C|-k)=\sum_{F \in \mathcal{F}}|F| ! \cdot(n-|F|) !-k \cdot n !$. Therefore,

$$
\sum_{F \in \mathcal{F}}|F| ! \cdot(n-|F|) !-k \cdot n ! \leqslant 0 .
$$

After rearranging, we obtain $\sum_{F \in \mathcal{F}}\left(\begin{array}{c}n \\ |F|\end{array}\right)^{-1} \leqslant k$, proving Theorem 4 .

Proof of Theorem 3. The statement of Theorem 3 is true for $k=2$ (base case) due to Theorem 2.

If neither $\varnothing$ nor $[n]$ are in $\mathcal{F}$, then we may apply Theorem 4 directly to obtain $|\mathcal{F}| \leqslant$ $\Sigma(n, k)$.

If both $\varnothing$ and $[n]$ are in $\mathcal{F}$, then $\mathcal{F} \backslash\{\varnothing,[n]\}$ is induced $Y_{k-1}$-free and induced $Y_{k-1}^{\prime}$-free. Therefore, it has size at most $\Sigma(n, k-1)$ by the induction hypothesis. Since $2+\Sigma(n, k-$ $1) \leqslant \Sigma(n, k)$ for $n \geqslant k+1$ and $k \geqslant 2$, we are done.

Now, without loss of generality, suppose that $\varnothing \in \mathcal{F}$ and $[n] \notin \mathcal{F}$. Consider the family $\mathcal{F}^{\prime}:=\mathcal{F} \backslash\{\varnothing\}$. By Theorem 4 , we have

$$
\sum_{F \in \mathcal{F}^{\prime}}\left(\begin{array}{c}
n \\
|F|
\end{array}\right)^{-1} \leqslant k
$$

and, by Lemma $7,\left|\mathcal{F}^{\prime}\right| \leqslant \Sigma(n, k)$.

Now suppose $\left|\mathcal{F}^{\prime}\right|=\Sigma(n, k)$. (Otherwise, we are done.) A consequence of the proof of Lemma 7 is that, in order for equality to hold in (3), the quantities $\left(\begin{array}{c}n \\ |F|\end{array}\right)$ (for $F$ in $\mathcal{F}^{\prime}$ ) must be as large as possible - that is, the sets $F \in \mathcal{F}^{\prime}$ must have size as close to $n / 2$ as possible. More precisely, in order for equality to hold in (3), the list of the quantities $\left(\begin{array}{c}n \\ |F|\end{array}\right)$ for $F \in \mathcal{F}^{\prime}$ in decreasing order (with multiplicities) must be the same as the list of the first $\Sigma(n, k)$ quantities $\left(\begin{array}{c}n \\ |S|\end{array}\right)$ for $S \subseteq 2^{[n]}$ in decreasing order (with multiplicities).

First, if $k$ and $n$ have different parities, then $\left|\mathcal{F}^{\prime}\right|=\Sigma(n, k)$ can only occur if

$$
\mathcal{F}^{\prime}=\left(\begin{array}{c}
{[n]} \\
\left\lfloor\frac{n-k}{2}\right\rfloor
\end{array}\right) \cup\left(\begin{array}{c}
{[n]} \\
\left\lfloor\frac{n-k}{2}\right\rfloor+1
\end{array}\right) \cup \cdots \cup\left(\begin{array}{c}
{[n]} \\
\left\lfloor\frac{n-k}{2}\right\rfloor+k-1
\end{array}\right) .
$$

However, in that case, $Y_{k-1}$ is an induced subposet of $\mathcal{F}^{\prime}$. Hence, adding $\varnothing$ produces an induced copy of $Y_{k}$ in $\mathcal{F}$, a contradiction. 
Second, if $k$ and $n$ have the same parity, then $\left|\mathcal{F}^{\prime}\right|=\Sigma(n, k)$ can only occur if $\mathcal{F}^{\prime}$ contains

$$
\left(\begin{array}{c}
{[n]} \\
\frac{n-k}{2}+1
\end{array}\right) \cup\left(\begin{array}{c}
{[n]} \\
\frac{n-k}{2}+2
\end{array}\right) \cup \cdots \cup\left(\begin{array}{c}
{[n]} \\
\frac{n-k}{2}+k-1
\end{array}\right)
$$

plus $\left(\begin{array}{c}n \\ \frac{n-k}{2}\end{array}\right)$ sets from $\left(\begin{array}{c}{[n]} \\ \frac{n-k}{2}\end{array}\right) \cup\left(\begin{array}{c}{[n]} \\ \frac{n-k}{2}+k\end{array}\right)$. If $\mathcal{F}^{\prime}$ contains any set from $\left(\begin{array}{c}{[n]} \\ \frac{n-k}{2}\end{array}\right)$, then it is easy to see that $Y_{k-1}$ is an induced subposet of $\mathcal{F}^{\prime}$ and adding $\varnothing$ produces an induced copy of $Y_{k}$ in $\mathcal{F}$. Otherwise, $\mathcal{F}^{\prime}$ must contain all of the sets from $\left(\frac{[n-k}{2}+k\right)$ and $n \geqslant k+2$. But in this case, $Y_{k-1}$ is again an induced subposet of $\mathcal{F}^{\prime}$, giving an induced copy of $Y_{k}$ in $\mathcal{F}$, again a contradiction.

Therefore, $\left|\mathcal{F}^{\prime}\right| \leqslant \Sigma(n, k)-1$, which implies $|\mathcal{F}| \leqslant \Sigma(n, k)$, as desired.

\section{Concluding Remarks}

Our approach to proving Theorem 3 amounts to a weighted double-counting where the choice of the charge values were motivated by a simple discharging argument. However, we believe charge functions that are motivated by more sophisticated discharging arguments, especially with the identification and enumeration of "spines," can lead to progress on other forbidden subposet problems.

We believe that a more general result than Theorem 3 holds. Recall that $Y_{k, r}$ denotes the $r$-fork with a $k$-shaft poset and $Y_{k, r}^{\prime}$ denotes its dual.

Conjecture 8. For all $k \geqslant 2$ and $r \geqslant 2$, there is an $n_{0}=n_{0}(k, r)$ such that if $n \geqslant n_{0}$, then $\operatorname{La}^{\sharp}\left(n,\left\{Y_{k, r}, Y_{k, r}^{\prime}\right\}\right)=\Sigma(n, k)$.

Theorem 3 is the case when $r=2$; note that for all $k \geqslant 2, n_{0}(k, 2)=k+1$.

\section{Acknowledgements}

During the preparation of this article, we learned that Tompkins and Wang recently proved Theorem 3 independently [18]. Their approach is very close to the method used in [13] and is very different from the approach introduced in this article.

The work for this project was done while Methuku was at Central European University and while Walker was at Iowa State University.

The authors thank reviewers of a previous incarnation of this manuscript and, most especially, thank Kirk Boyer, Kaave Hosseini, Eric Sullivan, and Casey Tompkins for many valuable discussions.

\section{References}

[1] E. Boehnlein and T. Jiang, Set families with a forbidden induced subposet, Combin. Probab. Comput., 21 (2012), no. 4, 496-511. 
[2] B. Bukh, Set families with a forbidden subposet, Electron. J. Combin., 16 (2009), no. 1 , \#R142.

[3] T. Carroll and G. O. H. Katona, Bounds on maximal families of sets not containing three sets with $A \cup B \subset C, A \not \subset B$, Order, 25 (2008), no. 3, 229-236.

[4] A. De Bonis and G. O. H. Katona, Largest families without an $r$-fork, Order, 24 (2007), no. 3, 181-191.

[5] A. De Bonis, G. O. H. Katona, and K. J. Swanepoel, Largest family without $A \cup B \subseteq$ $C \cap D$, J. Combin. Theory Ser. A, 111 (2005), no. 2, 331-336.

[6] P. Erdős, On a lemma of Littlewood and Offord, Bull. Amer. Math. Soc., 51 (1945), 898-902.

[7] J. R. Griggs and W.-T. Li, Progress on poset-free families of subsets. Recent Trends in Combinatorics, 317-338, IMA Vol. Math. Appl., 159, Springer, [Cham], 2016.

[8] J. R. Griggs, W.-T. Li, and L. Lu, Diamond-free families, J. Combin. Theory Ser. A, 119 (2012), no. 2, 310-322.

[9] J. R. Griggs and L. Lu, On families of subsets with a forbidden subposet, Combin. Probab. Comput., 18 (2009), no. 5, 738-748.

[10] D. Grósz, A. Methuku, and C. Tompkins, An upper bound on the size of diamond-free families of sets, J. Combin. Theory Ser. A, 156 (2018), 164-194.

[11] G. O. H. Katona, Forbidden intersection patterns in the families of subsets (introducing a method). Horizons of Combinatorics, 119-140, Bolyai Soc. Math. Stud., 17, Springer, Berlin, 2008.

[12] G. O. H. Katona and T. G. Tarján, Extremal problems with excluded subgraphs in the n-cube. Graph Theory (Eagów, 1981), 84-93, Lecture Notes in Math., 1018, Springer, Berlin, 1983.

[13] A. Methuku and C. Tompkins, Exact forbidden subposet results using chain decompositions of the cycle, Electron. J. Combin., 22 (2015), no. 4, \#P4.29.

[14] A. Methuku and D. Pálvölgyi, Forbidden hypermatrices imply general bounds on induced forbidden subposet problems. Combin. Probab. Comput., 26 (2017), no. 4, 593-602.

[15] E. Sperner, Ein Satz über Untermegen einer endlichen Menge, Math. Z., 27 (1928), no. $1,544-548$.

[16] H. T. Thanh, An extremal problem with excluded subposets in the Boolean lattice. Order, 15 (1998), no. 1, 51-57.

[17] C. Tompkins, Extremal problems on finite sets and posets (Doctoral dissertation), (2015) https://mathematics.ceu.edu/sites/mathematics.ceu.hu/files/ attachment/basicpage/27/tompkinsthesis.pdf Retrieved 29 September 2017.

[18] C. Tompkins and Y. Wang, On an extremal problem involving a pair of forbidden posets (arXiv preprint), (2017) arXiv:1710.10760. Retrieved 20 February 2018. 